\title{
Modal spectrum of permalloy disks excited by in-plane magnetic fields
}

\author{
I. Neudecker, ${ }^{1}$ K. Perzlmaier,${ }^{1}$ F. Hoffmann, ${ }^{1}$ G. Woltersdorf, ${ }^{1}$ M. Buess, ${ }^{2}$ D. Weiss, ${ }^{1}$ and C. H. Back ${ }^{1}$ \\ ${ }^{1}$ Institut für Experimentelle und Angewandte Physik, Universität Regensburg, Universitätsstrasse 31, 93040 Regensburg, Germany \\ ${ }^{2}$ Paul Scherrer Institut, CH-5232 Villigen PSI, Switzerland
}

(Received 27 December 2005; revised manuscript received 7 March 2006; published 27 April 2006)

\begin{abstract}
The mode spectrum of 4 micron sized ferromagnetic permalloy disks exhibiting a vortex structure in equilibrium is investigated in detail. Normal modes accessible by in-plane field excitations are examined. By means of spatially resolved techniques using Kerr microscopy we recorded the modal structure of the disks at zero bias field. We employed pulsed and continuous wave excitation. As a result we report on normal modes up to the fifth order. Using inductive and spatially resolved resonance measurements the evolution of the mode spectrum as a function of an external magnetic field was investigated. Quantized modes in longitudinal as well as in transverse direction in the uniform magnetized disks were observed. Good agreement between the experimental results and those from micromagnetic simulations was found.
\end{abstract}

DOI: 10.1103/PhysRevB.73.134426

PACS number(s): 75.75. $+\mathrm{a}, 75.30 . \mathrm{Ds}, 76.50 .+\mathrm{g}$

\section{INTRODUCTION}

The response of small magnetic elements to ultrashort magnetic field pulses and the accurate modelling of the spin excitations following a magnetic field pulse is a central question in advanced magnetic recording technology as switching times are further reduced and pushed well into the gyromagnetic regime. In recent years substantial effort has been put into the identification of the excitation spectrum of small ferromagnetic elements ${ }^{1-20}$ and into the investigation of their switching behavior in the precessional regime. ${ }^{21-23}$ While these problems can be addressed in the frequency domain using Brillouin light scattering (BLS) or ferromagnetic resonance (FMR) methods, $3,24,25$ time resolved scanning Kerr microscopy (TR-SKEM) experiments, ${ }^{4,12,26}$ or microfocus $\mathrm{BLS}^{20,27}$ now offer the opportunity to observe magnetic excitations on the picosecond time scale directly.

It is well known, that for moderate field pulse excitations and large magnetic elements (e.g., several tens of microns) the fundamental modes are essentially dominated by magnetostatic interactions. However, as the size of the element is reduced exchange dominated excitations start to play an essential role. Furthermore, even in large ferromagnetic elements exhibiting inhomogeneous internal fields exchange dominated excitations may be observed., ${ }^{9,14}$ The problem may be even more complicated for elements in the magnetostatic size regime; when higher order modes are excited the exchange interactions can no longer be ignored and the dynamic response gradually changes from a purely magnetostatic to an exchange dominated one. ${ }^{28}$ In this case the boundary conditions for the magnetization change continuously from quasipinned to unpinned conditions..$^{29-33}$

Here we report on the excitation spectrum of individual micron sized permalloy disks when excited by high frequency (hf) in-plane magnetic fields. In a first approach we imaged the magnetic response of the individual disks by recording a time resolved movie with high spatial resolution by means of TR-SKEM. In the Fourier transformation of the time domain image sequence we can identify several eigenmodes. These experiments were performed in zero bias field. In order to reveal the frequency spectrum of the disks as a function of a static in-plane magnetic bias field we perform ferromagnetic resonance (FMR) experiments using a vector network analyzer (VNA). VNA-FMR measurements differ from conventional FMR in one important point. Using a VNA one can sweep the frequency at a fixed external bias field while in conventional FMR the exciting hf frequency is fixed and the external field is swept through the resonance. For micron sized magnetic structures in a domain configuration this means that using VNA-FMR a spin configuration may be conserved at a fixed bias field and the resonance of this configuration is found by sweeping the frequency. In a third approach we combined the VNA-FMR setup with the optical TR-SKEM setup allowing for the spatially resolved investigation of individual eigenmodes of the system using continuous wave (cw) excitation. In contrast to the TRSKEM where a complicated superposition of modes is excited the spatial resolved ferromagnetic resonance scanning Kerr microscopy (FMR-SKEM) (Ref. 5) allows to image the spatial distribution of each individual eigenmode. In the regime of low field excitation, i.e., in a regime where the equation governing the precessional motion - the LandauLifshitz-Gilbert equation ${ }^{34}$ - can be linearized, the experiments in the time domain and in the frequency domain are complementary. Micromagnetic simulations based on the LLG code $^{35}$ back-up our experimental findings.

\section{SAMPLE PREPARATION AND CHARACTERIZATION}

The sample studied experimentally is an array of 1 $\times 100$ ferromagnetic permalloy $\left(\mathrm{Ni}_{81} \mathrm{Fe}_{19}\right)$ disks with a thickness of $20 \mathrm{~nm}$ and a diameter of $4 \mu \mathrm{m}$ fabricated by $e$-beam lithography and lift-off technique. The edge to edge distance of 2 micron prevents interdisk coupling. For corrosion protection the disks were capped with $2 \mathrm{~nm} \mathrm{Al}$. We used x-ray fluorescence to obtain the sample thickness on a wafer prepared at the same time as the actual samples. On the same wafer we determined the magnetic moment of the permalloy samples using a superconducting quantum interference device (SQUID) magnetometer. We obtain $\mu_{0} M_{\mathrm{s}}=1.0 \mathrm{~T}$ and thickness $t=20 \mathrm{~nm}$. The elements were placed on top of a 
a)

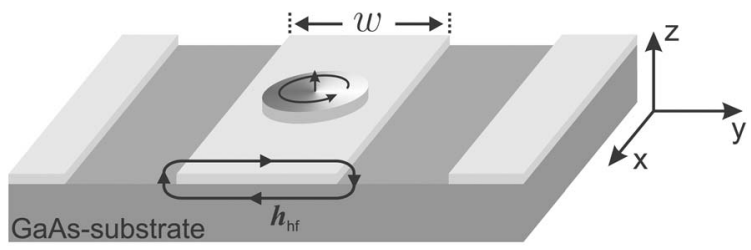

b)

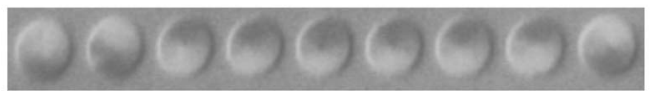

FIG. 1. The permalloy disk on top of the coplanar waveguide. The schematic sketch in (a) shows the vortex state with the magnetization curling in the plane of the disk around the perpendicular component at the center. Photoemission electron microscopy investigations confirmed the equilibrium ground state of the disks to be a vortex (b). The black/white contrast represents the $y$ component of the magnetization $\boldsymbol{M}$.

$350 \mathrm{~nm}$ thick coplanar waveguide fabricated onto semiinsulating GaAs substrate using standard optical lithography and subsequent lift-off technique. The impedance of the waveguide with a central conductor width of $w=10 \mu \mathrm{m}$ [see Fig. 1(a)] is matched to $50 \Omega$.

\section{EXPERIMENTAL SETUPS}

For the investigation of the modal spectrum of the disks three different experimental setups were employed, which are explained in this section.

(i) In our conventional TR-SKEM experiments the ground state of the magnetic elements is disturbed by a short and weak - typical field strengths do not exceed $1.0 \mathrm{mT}-$ magnetic tipping pulse $\boldsymbol{h}_{\text {pulse }}$ pointing along the $y$ direction [see Fig. 1(a)]. The precessional motion of the spins is excited and one is left to observe the response on a macroscopic length scale given by our optical experiment with approximately $300 \mathrm{~nm}$ spatial resolution. ${ }^{4}$ Polar Kerr images are obtained as a function of time delay between application of the tipping pulse and the probe pulse in a stroboscopic experiment. Further details of the experiment are described in Ref. 4.

(ii) Inductive measurements were carried out by employing ferromagnetic resonance (FMR) using a vector network analyzer (VNA). In doing so the VNA serves both as the source and as the detector of the sinusoidal hf signal. The resonance frequency is found by measuring the absorption of the sample as a function of the frequency of the exciting signal. The frequency range of our network analyzer is $45 \mathrm{MHz}$ to $20 \mathrm{GHz}$. Details concerning the inductive setup will be presented in Ref. 36.

(iii) Spatially resolved ferromagnetic resonance (FMR) investigations were realized by combining the VNA-FMR setup with the TR-SKEM setup - hence FMR-SKEM. A sketch of the setup is shown in Fig. 2. The cw hf signal of the network analyzer at a constant frequency is used for exciting the magnetic system. In order to ensure fixed correlation between the phase of the excitation and the probe beam, the Ti:sapphire oscillator is locked to $80 \mathrm{MHz}$. The VNA in turn is locked to this clock by $80 / 8 \mathrm{MHz}=10 \mathrm{MHz}$ (see inset in

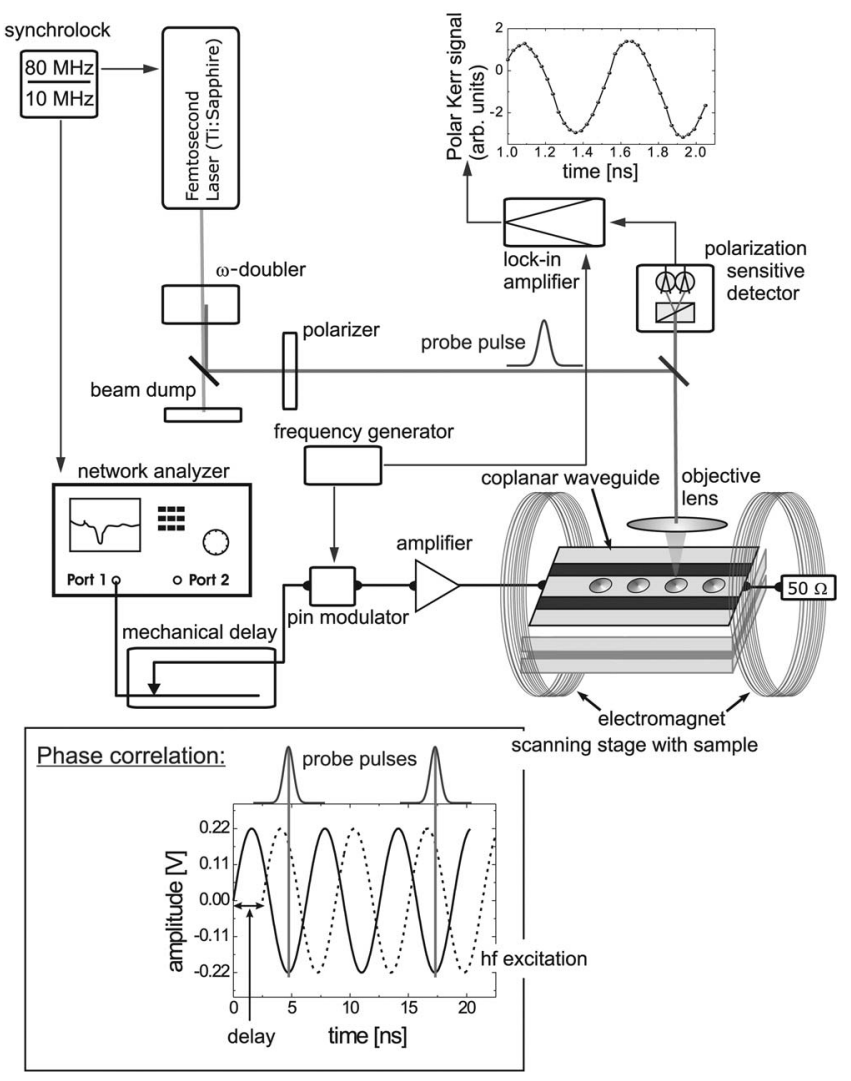

FIG. 2. The spatially resolved ferromagnetic resonance (FMRSKEM) setup. After frequency doubling the pulses of a mode locked Ti:sapphire laser system are used to record the magnetic response by means of magneto-optical Kerr effect. The hf signal from the network analyzer excites the disks on top of the coplanar waveguide. A mechanical delay allows for investigating the response of the sample at any phase of the exciting cw high frequency field (see inset bottom).

Fig. 2). As a result the minimum step for sweeping the frequency is given by $80 \mathrm{MHz}$, the repetition rate of the laser. In order to investigate the magnetic response at any specific phase of the excitation, the hf signal is guided to the coplanar waveguide via a mechanical trombone delay generator. A pin modulator acts as a chopper for the hf signal to allow lock-in detection. It is important to note that by employing FMRSKEM the dynamic response is measured at a fixed phase, so that the phase information is now hidden in the experimentally determined parameters. The resulting images correspond to the combination of amplitude and phase of the Fourier transformed pulsed experiments or simulations. The spatial resolution is still given by the Kerr setup of approximately $300 \mathrm{~nm}$. In addition to the modal structure at a specific frequency the setup allows to measure resonance lines as a function of the position on the sample. To do so the laser spot is left at one point on the sample while scanning the frequency of the cw excitation. At every frequency the phase of the excitation is adjusted with respect to maximum amplitude of the Kerr signal. The resonance frequency can be determined by measuring the Kerr rotation as a function of the excitation frequency. 


\section{ENERGETICS AND MICROMAGNETICS}

We study disk shaped permalloy ferromagnetic elements in a regime where the ratio of disk diameter to thickness leads to a vortex ground state. ${ }^{37-39}$ In order to reduce the magnetic stray field energy, the magnetization vector of the element lies in the plane of the disk and curls around the center. As a consequence of this particular geometry the spins in the center of the element must assume a perpendicular orientation [see Fig. 1(a)]. The direction of flux closure and of the center spin lead to four different possible magnetization configurations. However, considering the symmetries of the four cases, they reduce to two chiralities. The magnetization distribution leads to a homogeneous internal field distribution within the flux closed state with substantial deviations only at the edge of the element and at the vortex core where the effect of the exchange energy is clearly noticeable. A sketch of the vortex ground state together with an $\mathrm{X}$-ray photoemission electron microscope image using the circular magnetic dichroism obtained at the SIM beam line of the Swiss Light Source is shown in Figs. 1(a) and 1(b), respectively. The normal mode spectrum of ferromagnetic elements is governed by the total internal field $\boldsymbol{H}_{\text {eff }}$ and the relevant boundary conditions. In this size and thickness regime it is justified to use purely dipolar boundary conditions to describe the low-lying normal eigenmodes. ${ }^{40,41}$ In contrast in micromagnetic simulations the behavior of the magnetic moments at the boundaries is naturally described by Maxwell's equations. A more thorough analysis of the normal mode spectrum of flux closed ferromagnetic disks can be found in Refs. 42-44 and references therein.

The total internal field $\boldsymbol{H}_{\text {eff }}$ results from the functional derivative of the total energy density $\varepsilon_{\text {tot }}$ with respect to the reduced magnetization $\boldsymbol{m}(\boldsymbol{r})=\boldsymbol{M}(\boldsymbol{r}) / M_{s}, M_{s}$ being the constant saturation magnetization,

$$
\boldsymbol{H}_{\mathrm{eff}}=-\frac{1}{\mu_{0} M_{s}} \frac{\delta \varepsilon_{\mathrm{tot}}}{\delta m},
$$

where $\mu_{0}$ is the permeability of free space. For small angle deviations from the ground state the total internal field $\boldsymbol{H}_{\text {eff }}$ dictates the precessional response of a magnetic element as described by the Landau-Lifshitz-Gilbert equation, ${ }^{34}$

$$
\frac{d}{d t} \boldsymbol{M}=-\gamma_{0} \mu_{0}\left(\boldsymbol{M} \times \boldsymbol{H}_{\mathrm{eff}}\right)+\frac{\alpha}{M_{s}}\left(\boldsymbol{M} \times \frac{d}{d t} \boldsymbol{M}\right) .
$$

Here, $\gamma_{0}=g \mu_{B} / \hbar$ is the gyromagnetic ratio and $\alpha$ the damping parameter. In our case, the effective field comprises the exchange field, the demagnetization field, and the external bias field $\boldsymbol{H}_{\text {bias }}$ applied along the $x$ direction. The small anisotropy field of permalloy is ignored in the following and the external exciting field serves merely as a tipping hf field. In the micromagnetic simulations, the disks exhibiting a vortex ground state are subjected to a short field pulse $\boldsymbol{h}_{\text {pulse }}$ applied along the $y$ direction. For the simulations we used an instantaneous $0.5 \mathrm{mT}$ amplitude field pulse with a duration of $50 \mathrm{ps}$. The magnetic properties of the permalloy disks used in the simulations are as follows: Exchange constant $A=13 \times 10^{-12} \mathrm{~J} / \mathrm{m}$, anisotropy constant $K_{u}=0$, saturation magnetization $\mu_{0} M_{s}=1 \mathrm{~T}$, and damping constant $\alpha=0.008$.
Micromagnetic simulations were performed using the LLG code $^{35}$ unless stated otherwise, on a $5 \mathrm{~nm}$ and $15 \mathrm{~nm}$ grid for disks with and without vortex core, respectively. The equations of motion were integrated in $0.5 \mathrm{ps}$ steps from $t=0$ to $5 \mathrm{~ns}$. The data were stored for the entire array and for the average value of each magnetization component. Edge corrections were applied to the boundary of the disk. For finding the frequencies of the different modes we perform global Fourier transformation of the averaged time domain data. In order to gain a deeper insight into the structure of the excited modes we transform the time domain output from simulation into the frequency domain by employing a phase-sensitive local Fourier transformation procedure described in detail in Refs. 6 and 45. For each pixel the time domain data stream is Fourier transformed and subsequently reassembled in order to obtain the amplitude and phase distribution across the magnetic object as a function of frequency. Moreover the results from simulations are filtered by means of a $300 \mathrm{~nm}$ - the spatial resolution of the optical setup - twodimensional Gaussian window in order to compare them to the experimental data.

\section{RESULTS AND DISCUSSION}

Figure 3 shows the $z$ component of the magnetization at $H_{\text {bias }}=0$ averaged over one half of the disk as a function of time elapsed after application of the tipping field pulse. In Fig. 3(a) we show the experimental data obtained from a 3.4 ns long image sequence, Fig. 3(b) shows the results from the simulation. In both cases one immediately realizes that more than a single frequency is responsible for the time evolution. Fourier transformation (FT) into the frequency domain confirms this finding and shows clear peaks at $2.6 \mathrm{GHz}$ (2.68 GHz and $2.83 \mathrm{GHz}), 4.0 \mathrm{GHz}(4.27$ and $4.47 \mathrm{GHz})$, and $5.4 \mathrm{GHz}(5.42$ and $5.61 \mathrm{GHz})$ for the experimental (simulated) results [see Figs. 3(c) and 3(d)]. Note that in this enumeration only peaks, which are found both in experiment and simulation have been considered. Zero-filling and a Hamming cutoff window were used in the FT. We notice at this point, that all peaks in the micromagnetic simulation show a doublet structure and will come back to this point when locally analyzing the Fourier transformed data.

When zooming into the central region of the element in our micromagnetic simulation we observe the vortex core precessing around the center of the disk with a revolution time of approximately $14 \mathrm{~ns}$, which corresponds to a frequency of $70 \mathrm{MHz}$. This gyrotropic oscillation of the vortex core was described in detail in Ref. 46 and was observed experimentally in Refs. 12, 16, and 47. The maximum scan length of our experimental time delay is $3.4 \mathrm{~ns}$, which corresponds to a resolution of approximately $0.3 \mathrm{GHz}$ in the Fourier transformed data. As a consequence we are not able to detect the gyrotropic mode in our experiment.

In order to analyze the excited modes in greater detail, we perform a local Fourier transformation of the data as already explained above. In Fig. 4 we plot the local Fourier transformation of the $z$ component of the magnetization [from experiment, Fig. 4(a) and from simulation, Fig. 4(b)]. In our Kerr microscopy experiments we use the polar configuration 
Experiment:
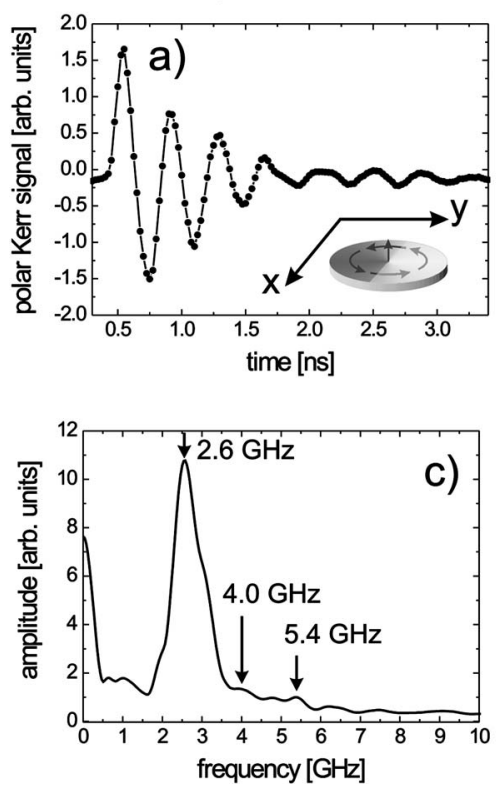

Simulation:
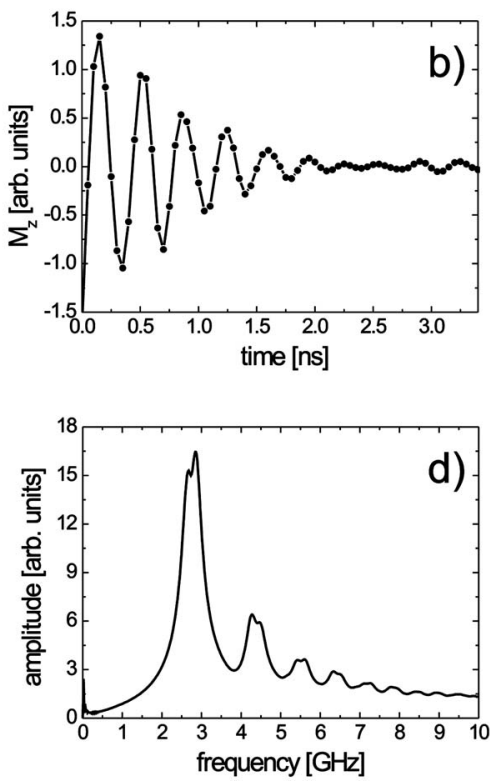

FIG. 3. The $z$ component of the magnetization $\mathbf{M}$ as a function of time obtained from timeresolved magneto-optic Kerr microscopy (a) and from micromagnetic simulation (b) averaged over one half of the disk [see inset in (a)]. The corresponding Fourier transform of the experimental and the simulated data is shown in (c) and (d), respectively. and hence only detect the $z$ component of the magnetization. The corresponding modal structure is reminiscent of Bessel functions of first order, ${ }^{17,40}$ but shows an additional node in the azimuthal direction caused by the asymmetry of the torque due to the in-plane field pulse excitation. For a field pulse applied along the $y$ direction, the two sides of the disk experience an opposite initial torque. This phase shift by $\pi$ can be observed in the phase of the modes. We label the modes using two numbers $n$ and $m$ according to the number of radial $(n)$ and azimuthal $(m)$ nodes. Correspondingly, the modes in zero bias field depicted in Fig. 4 are labeled

\section{a) Experiment:}

b) Simulation:
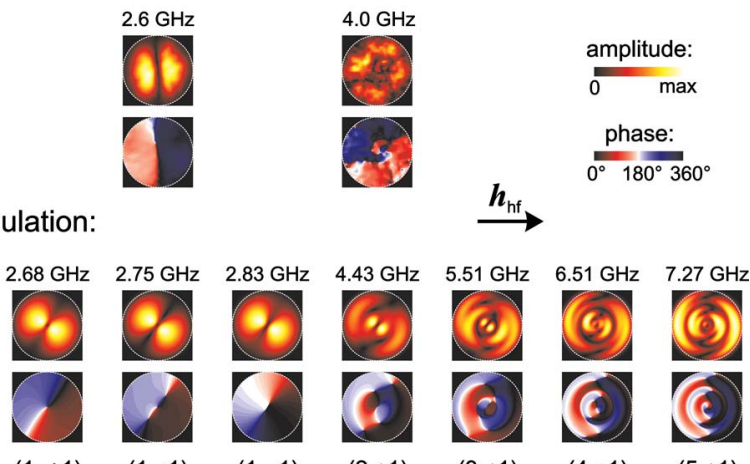

FIG. 4. (Color online) Amplitude (upper row) and phase (lower row) generated by local Fourier transform of the time domain data obtained from TR-SKEM (a) and micromagnetic simulations (b) at zero bias field. The modes are labeled by $(n, m)$ according to their number of radial $(n)$ and azimuthal $(m=1)$ nodes. Both peaks of the doublet structure observed in the global FT from simulation in Fig. 3 are exemplarily shown for the fundamental mode $(1, \pm 1)$. The phase of mode $(1,+1)$ and mode $(1,-1)$ seems to circulate clockwise and counterclockwise around the center, respectively. In addition the superposition is shown, which was found at the frequency between the two modes. For mode $(2,1)$ to mode $(5,1)$ only the superpositions $( \pm$ mode) of the doublet structures are shown. $(n, m=1)$. For these normal modes the frequency increases with increasing radial node number as shown in Ref. 41, where we used a simple model based on purely dipolar excitations. In a more sophisticated model Zivieri and Nizzoli ${ }^{44}$ calculated the modal spectrum of cylindrical dots by taking into account the influence of both the surface and volume dynamic exchange interaction and dipolar fields. Here, we limit ourselves to the comparison to micromagnetic simulations. In the simulations, shown in Fig. 4(b), we also observe Bessel function like modes $(n, m=1)$.

When analyzing the local FT obtained from the raw image series from simulation with respect to the doublet structure observed in the global FT [see Fig. 3(d)], we find that not only one clear mode with $(n=1, m=1)$ is found. Indeed, a second mode is observed in the local FT which shows nearly equal features, exemplarily depicted for mode $(1,+1)$ and mode $(1,-1)$ in Fig. 4(b). However, these pairs of modes differ in their phase. While the phase of mode $(1,+1)$ seems to circulate clockwise, for mode $(1,-1)$ it seems to circulate counterclockwise around the disk center. The splitting of the azimuthal modes, which has already been observed experimentally in Refs. 18 and 48 has recently been explained by Ivanov and Zaspel. ${ }^{43}$ According to these authors the vortex core breaks the azimuthal symmetry of the disk. Coupling between the vortex core and the magnetostatic spin waves lifts the degeneracy of the clockwise and counterclockwise travelling magnons. As a consequence of the frequency splitting the two magnons circulate with different velocities around the disk center. Therefore, their superposition, namely mode $(1, \pm 1)$, appears to be tilted with respect to the direction of the exciting pulse. ${ }^{48}$ Although we observe a very broad peak in the experiment with a clear shoulder at $3.0 \mathrm{GHz}$ in the global FT of the fundamental mode $(1,1)$ [see Fig. 3(c)], in the local Fourier transform we do not observe a second mode with opposite circulating phase. This might come from imperfections of the real sample, which constitute pinning centers for the vortex core. Hence, the gyrotropic 


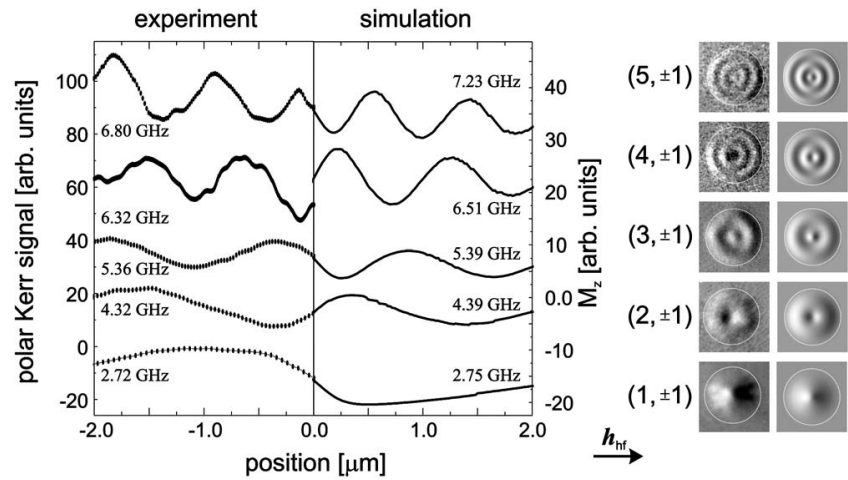

FIG. 5. The images show the Kerr rotation and the $z$ component of $\mathbf{M}$ obtained from experiment (FMR-SKEM) (left row) and simulation (right row), respectively. Line scans across the disk center parallel to $\boldsymbol{h}_{\mathrm{hf}}$ reveal the modal structure obtained from experiment (from -2.0 to $0.0 \mu \mathrm{m}$ ) and from simulation (from 0.0 to $2.0 \mu \mathrm{m}$ ). The modes are labeled by $(n, m=1)$ according to their number of radial $(n)$ and azimuthal $(m)$ nodes. The black/white contrast represents amplitude combined with phase.

motion is disturbed and the interaction with the normal modes is attenuated. Moreover, the splitting of the doublet structure is supposed to be of the order of the vortex gyrotropic frequency, which is with $70 \mathrm{MHz}$ rather small for the investigated disks. Also in Ref. 48 the influence of the vortex core motion could not be observed for disks with diameter greater than $3 \mu \mathrm{m}$.

An alternative experimental approach to investigate the modal spectrum of the disks is to exclusively excite the different modes by means of a continuous wave excitation. ${ }^{5,49}$ This can be realized by combining the resonant excitation with our magneto-optical detection scheme as described above (see Fig. 2). An advantage of exclusively exciting single modes instead of triggering a complicated superposition of modes is the possibility to be able to observe higher order modes. Due to the strong decay of the overall signal using pulsed excitation only modes $(n=1, m=1)$ and $(n=2$, $m=1$ ) can be extracted from our experimental data [see Fig. 4(a)]. However, by means of $\mathrm{cw}$ excitation we are indeed able to detect the normal modes $(n, m=1)$ up to $n=5$. In Fig. 5 we show the normal modes up to the fifth order obtained from FMR-SKEM measurements. The frequencies of the modes are in good agreement with the pulsed experiment and the simulations. Note, that due to the $\mathrm{cw}$ excitation the black/ white contrast of the obtained images encodes amplitude combined with the phase of the modes. We now combine amplitude $A$ and phase $\varphi$ by $A \sin \left(\varphi+\varphi_{0}\right)$ also for the simulated data in order to compare them to data from FMRSKEM. We use $\varphi_{0}$ as a phase offset to adjust maximum contrast. In order to reveal the detailed modal structure we performed line scans across the disk center parallel to the $\mathrm{hf}$ field $\boldsymbol{h}_{\mathrm{hf}}$, and found excellent agreement with the simulated data, see Fig. 5.

We will now follow the evolution of the modes as a function of a bias field applied along the $x$ direction, perpendicular to the hf field. Due to the highest intensity of mode ( $n$ $=1, m=1$ ) we do so by observing this mode by means of the inductive VNA-FMR technique (see Fig. 6). Figure 6(a)
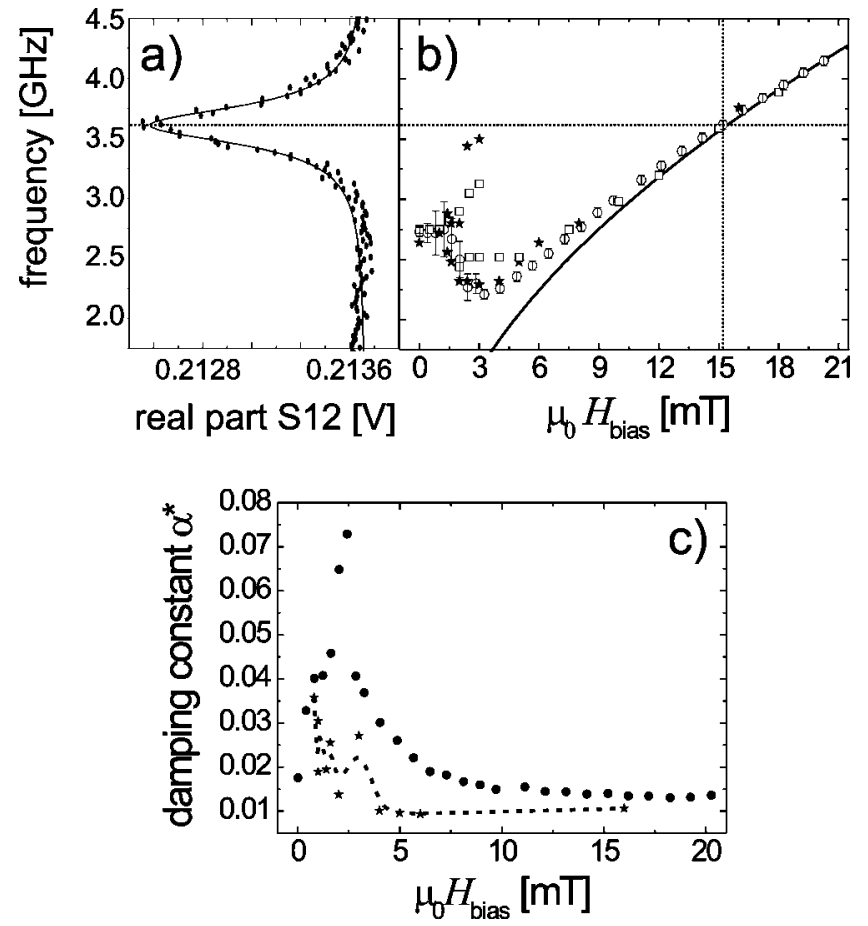

FIG. 6. Raw data from VNA-FMR measurements (dots) and the fit to a Lorentzian line (a). The extracted frequency of the fundamental mode $(1,1)$ as a function of the bias field (b) obtained by VNA-FMR measurements (open circles), by micromagnetic simulations carried out using OOMMF (open squares) and by frequency sweeps using FMR-SKEM measurements (stars). The data points were obtained from an up-sweep (increasing the bias field starting from zero field). The fit to the Kittel equation is represented by the solid line in (b). The effective damping parameter $\alpha^{*}$ shown in (c), was extracted from VNA-FMR (dots) and FMR-SKEM (stars) measurements.

shows raw data from a VNA-FMR measurement with a resonance peak at $f=3.6 \mathrm{GHz}$ with a corresponding bias field of $\mu_{0} H_{\text {bias }}=15 \mathrm{mT}$. The frequency and the peak width were extracted by fitting the peak to a Lorentzian line shape. By performing this procedure to data recorded at different bias fields we obtain the frequencies and linewidths as a function of the external field as shown in Fig. 6(b). It can be seen, that the frequency of mode $(1,1)$ [open circles in Fig. 6(b)] decreases from its initial value of $2.7 \mathrm{GHz}$ to about $2.2 \mathrm{GHz}$ at a bias field of $\sim 3.0 \mathrm{mT}$. It then starts to increase again and merges with the behavior expected from the Kittel formula [solid line in Fig. 6(b)] corrected for the demagnetizing factor $N$ corresponding to the aspect ratio of the investigated disks:

$$
f=\frac{\mu_{0} \gamma}{2 \pi} \sqrt{H_{\text {bias }}\left(H_{\text {bias }}+N M_{s}\right)},
$$

where the gyromagnetic ratio is $|\gamma|=g \times 97.9 \mathrm{GHz} / \mathrm{T}$. For the investigated dots the demagnetizing factor $N$ is $0.97 .{ }^{50}$ By means of SQUID magnetometry the saturation magnetization was determined to be $\mu_{0} M_{s}=1.0 \mathrm{~T}$. The $g$ factor was allowed to vary and was used as a fitting parameter resulting in $g=2.09$, which is in good agreement with values from the 
literature. ${ }^{51}$ For the single domain disk (for $\mu_{0} H_{\text {bias }}$ $\geqslant 4.0 \mathrm{mT}$ the vortex is expelled) this behavior is expected as soon as the spins are mostly aligned along the field direction. In fact, at higher field values, the behavior is well described by the Kittel formula. From the linewidth $\Delta f$ (half-width at half-maximum) of the measured resonance curves one can determine the effective damping parameter by using $\alpha^{*}$ $=4 \pi \Delta f /\left[\gamma \mu_{0}\left(2 H_{\text {bias }}+N M_{s}\right)\right]$. The resulting effective damping parameter extracted from VNA-FMR (dots) and FMRSKEM (stars) measurements is shown in Fig. 6(c). We observe a clear peak at $2.5 \mathrm{mT}$ in the data from VNA-FMR, which might result from the integrative nature of the technique, which means that the average signal of the whole array of 100 disks is measured. Since the vortex displacement as a function of bias field might be different for each disk one measures various resonance curves at different frequencies. As a consequence, the linewidth of the resulting resonance curve is much wider than it would be for a single disk. This interpretation is confirmed by the effective damping from FMR-SKEM measurements recorded at one single disk, which do not show a clear peak [see stars in Fig. 6(c)]. The low signal-to-noise ratio (SNR) of the VNA-FMR in comparison to the optical setup allows only to investigate the fundamental mode $(1,1)$. However, in the simulations carried out using the object oriented micromagnetic framework ${ }^{52}$ (OOMMF) and in the optical measurements we are able to observe the modal spectrum in more detail. In the intermediate region when the vortex has not left the disk yet $\left(\mu_{0} H_{\text {bias }}<4.0 \mathrm{mT}\right)$, the mode observed in the VNA measurements is actually a superposition of two modes. In Fig. 6(b) the open squares correspond to simulations and stars correspond to data obtained from frequency scans using FMRSKEM.

By moving the vortex to the side the two large regions with maximum torque now differ in size. As the modes are originally dominated by the dipolar interactions the change of size immediately leads to a difference in the modal frequencies, with the frequency of the smaller domain increasing and the one of the larger domain slightly decreasing. When the vortex is further displaced, the contribution to the exchange energy within the smaller domain dominates and the frequency increase becomes larger. Eventually, the frequency of the mode in the domain which is growing as a function of bias field merges with the quasiuniform mode of the single domain case. Figure 7 shows in detail the evolution of the two coexisting modes as a function of the bias field obtained by spatially resolved frequency scans using the resonant Kerr technique (stars in Fig. 7). Moreover, images of the modal structure shown in Fig. 7 confirm the described behavior. Due to forced oscillation inside the domain which is not at resonance with the exciting field the images in Fig. 7 show amplitude distribution in both domains. However, as soon as the vortex is expelled from the disk, above the annihilation field of $\mu_{0} H_{\text {bias }}=4.0 \mathrm{mT}$ the mode located in the shrinking region disappears and the one located in the growing domain shows quasiuniform precession (see image in the lower right-hand corner in Fig. 7).

Due to the disappearance of the vortex core the element assumes a quasi single domain state, so that the magnetization is now pointing along the $x$ direction leading to mag-

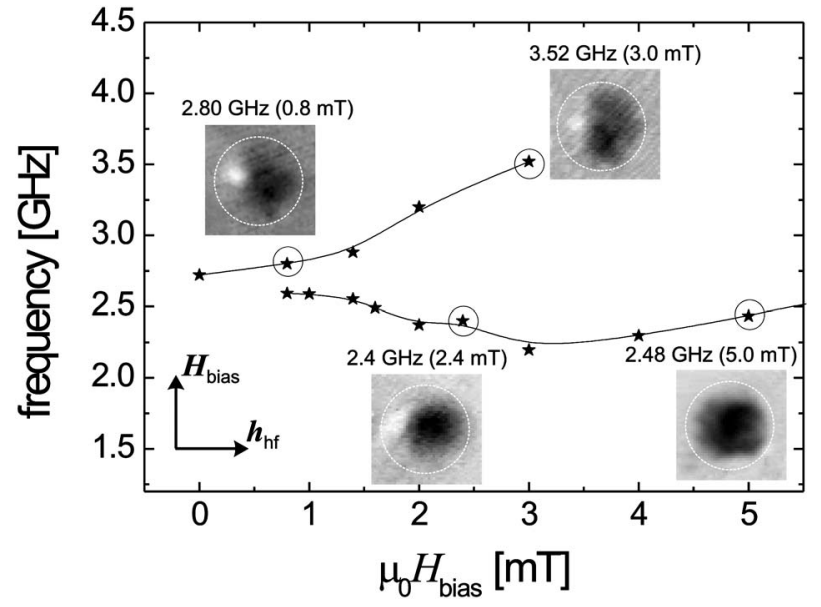

FIG. 7. The transition region from vortex to single domain state where the coexistence of two modes with different dispersion was observed. Data points were obtained from frequency scans using FMR-SKEM for an up-sweep. The images of the modal structure recorded by means of FMR-SKEM correspond to the encircled data points.

netic poles at the edges of the disk. The resulting stray field in turn locally softens the effective internal field of the system. This effect has first been observed by Hiebert et al. ${ }^{1}$ The resulting internal effective field for the entire disk (a) and as a line-scan across the disk center parallel to the external field (b) for $\mu_{0} H_{\text {bias }}=20 \mathrm{mT}$ are depicted in Fig. 8. Due to the localized inhomogeneity of the internal field near the edges two regions inside the disk can be distinguished with respect to the effective field. A quasi homogeneous region around the center of the disk and a strongly inhomogeneous region around the disk edges. Furthermore, in contrast to a rectangular slab the internal field of the disk depends also on the $y$ direction (perpendicular to the applied field) due to the curvature of its boundary.

First we focus on the homogeneous region near the disk center. We investigated the modal spectrum of this area at $\mu_{0} H_{\text {bias }}=5 \mathrm{mT}$ by means of spatially resolved FMR-SKEM measurements and micromagnetic simulations shown in Fig. a)

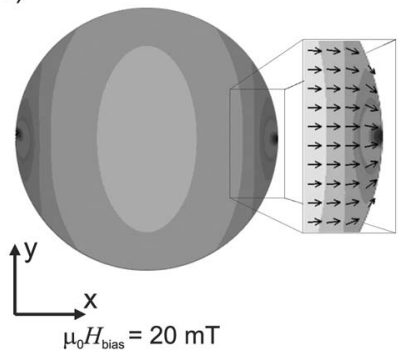

b)

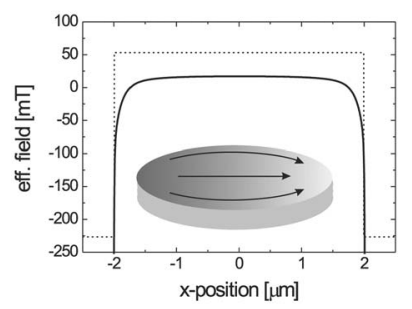

FIG. 8. The effective internal field of the entire disk encoded by a black/white contrast at $\mu_{0} H_{\text {bias }}=20 \mathrm{mT}$ (a). In order to relate the static magnetization configuration $\mathbf{M}$ (represented by the arrows) to the effective field a small area of the edge region is enlarged. A line scan along the external bias field reveals the field profile as a function of the $x$ position (b). All data was extracted from micromagnetic simulation. 
a) Experiment:

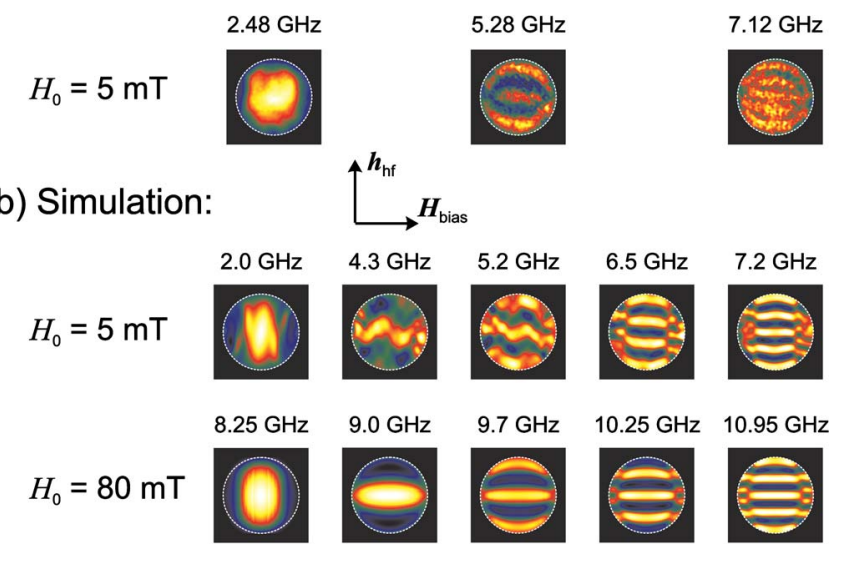

FIG. 9. (Color online) Spatially resolved images from FMRSKEM measurements (a) and from micromagnetic simulations (b) at $\mu_{0} H_{\text {bias }}=5.0 \mathrm{mT}$ and $\mu_{0} H_{\text {bias }}=80.0 \mathrm{mT}$. The simulated data was obtained by local Fourier transformation of the time domain results.

9(a) and the upper part of Fig. 9(b), respectively. At $2.48 \mathrm{GHz}(1.9 \mathrm{GHz})$ in the experimental (simulated) data we observe a mode with amplitude distribution throughout the entire region of quasihomogeneous internal field [see Fig. 8(a) ]. At $f=5.28 \mathrm{GHz}(5.2 \mathrm{GHz})$ and $7.12 \mathrm{GHz}(7.2 \mathrm{GHz})$ we exemplarily observe in experiment (simulation) modes with four and eight nodal lines, respectively, parallel to the applied field $\boldsymbol{H}_{\text {bias }}$. We note at this point that also the modes with 2 and 6 nodal lines should be observable in the experiment. However with our SNR we could not detect these modes. Microfocus BLS should be able to reveal also these modes on account of its higher SNR. ${ }^{20,27}$ Consequently these modes are quantized transverse to the bias field and to the static magnetization. Since in addition the mode frequency increases with increasing node number these modes are reminiscent of Damon-Eshbach-type modes. ${ }^{53}$ The modes observed in the simulations at $\mu_{0} H_{\text {bias }}=5 \mathrm{mT}$ are disturbed with respect to the orientation of the their nodal lines [see upper part of Fig. 9(b)]. However, simulations carried out at $\mu_{0} H_{\text {bias }}=80 \mathrm{mT}$ [see lower part of Fig. 9(b)] show the same modes without any deviation. Therefore, we attribute the deviation to arise from the inhomogeneous magnetization configuration even in the center region due to the moderate bias field of $\mu_{0} H_{\text {bias }}=5 \mathrm{mT}$. The mode images obtained by the experiment do not show these disturbances. This might be due to the fact that the vortex annihilation field of the actual samples is lower than in the simulations, leading to a more homogeneous magnetization configuration.

Now we concentrate on the edge region showing a strong inhomogeneous internal field as depicted in Fig. 8. Spatially resolved images from FMR-SKEM measurements reveal that in addition to the modes in the center, localized modes are found in the edge region [see Fig. 10(a)]. In Fig. 10(a) we show the modal structures as a function of the external bias field. The data points were determined by performing spatially resolved frequency scans using FMR-SKEM. While the upper images show the quasiuniform fundamental mode of the center region, we observe another mode localized in the edge region of the disk, see lower images in Fig. 10(a).
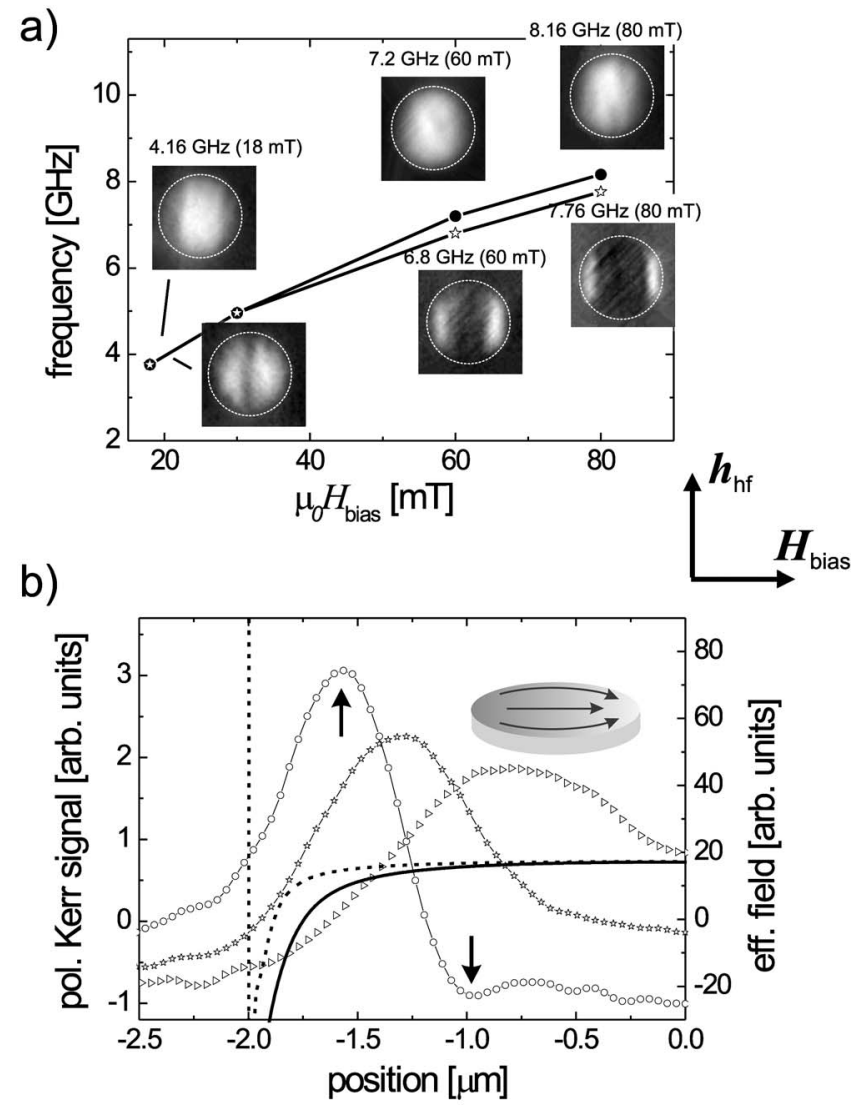

FIG. 10. The quasiuniform (filled stars) and the localized edge modes (open stars) as a function of the bias field obtained from frequency scans using FMR-SKEM (a). Corresponding images reveal the structure of the different modes. While the center and the edge mode are degenerate below $30 \mathrm{mT}$, the different dispersion relations lead to a splitting in frequency above $60 \mathrm{mT}$. Horizontal line scans for the edge modes across the center of the disk for $4.16 \mathrm{GHz}$ (triangles), $6.8 \mathrm{GHz}$ (stars), and $7.76 \mathrm{GHz}$ (dots) are shown in (b). The effective field at $\mu_{0} H_{\text {bias }}=20 \mathrm{mT}$ and $80 \mathrm{mT}$ obtained from micromagnetic simulations is represented by the solid and dashed curve, respectively. The vertical dotted line marks the left boundary of the disk.

Their spatial distribution is reminiscent of localized edge modes $^{6,9,10}$ caused by the inhomogeneous internal field within the disk which leads to a "well" for magnetic excitations. ${ }^{14,54}$ In the experiment the quasiuniform mode in the disk center and the localized edge mode are observed at the same frequency up to an external bias field of $30 \mathrm{mT}$. They can be distinguished as their maximum amplitudes are found at different phases. However, by increasing the bias field, the different dispersion relations cause a splitting in frequency of the two modes. This effect can be observed experimentally above $\mu_{0} H_{\text {bias }}=60 \mathrm{mT}$ [see Fig. 10(a)]. At the maximum bias field of $80 \mathrm{mT}$ applied in the experiment the frequency splitting is $\Delta f=400 \mathrm{MHz}$.

In order to investigate the spatial distribution of the edge modes as a function of the external bias field we performed line-scans across the disk center parallel to the bias field [see Fig. 10(b)]. Furthermore, the effective field profile of the disk is plotted in Fig. 10(b) for $\mu_{0} H_{\text {bias }}=20 \mathrm{mT}$ (solid line) 


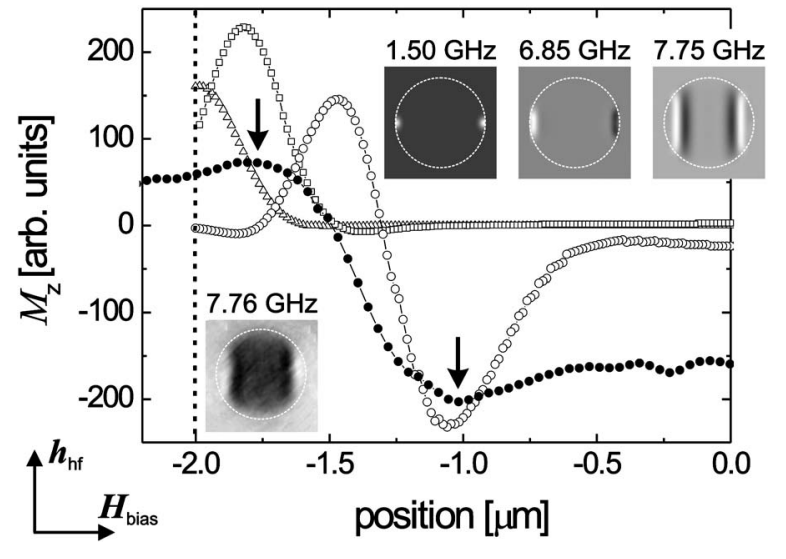

FIG. 11. Images and line scans from experiment (lower left inset and solid dots) and from simulation (upper right insets and open symbols) depict the amplitude distribution of the localized edge modes at $\mu_{0} H_{\text {bias }}=80 \mathrm{mT}$. The line scans are performed across the center of the disk parallel to the bias field. Open symbols showing the amplitude distribution for $f=1.5 \mathrm{GHz}$ (triangles), $f=6.85 \mathrm{GHz}$ (squares), and $f=7.75 \mathrm{GHz}$ (dots) represent simulation. The nodes which can be clearly seen in the data from simulation $(f=7.75 \mathrm{GHz})$ are also indicated in the data from experiment (see arrows). The vertical dotted line marks the left boundary of the disk. Images from simulations were obtained by local Fourier transformation of the time domain data.

and $\mu_{0} H_{\text {bias }}=80 \mathrm{mT}$ (dashed line). We found the amplitude distribution to become narrower with increasing bias field, which is caused by a corresponding decrease of the inhomogeneous edge region. ${ }^{14,55}$

In order to back-up our experimental results, micromagnetic simulations were carried out at $\mu_{0} H_{\text {bias }}=80 \mathrm{mT}$. Figure 11 shows the images and the corresponding line scans, which reveal three quantized modes localized in the edge region. This observation meets expectations, because when the "well" becomes narrower, also higher order edge localized modes should appear. ${ }^{54}$ However, in the experiment we could not detect additional nodes for the edge modes below $\mu_{0} H_{\text {bias }}=80 \mathrm{mT}$. In fact for the edge mode at $80 \mathrm{mT}$ we observe an antinode indicated at position $-1.0 \mu \mathrm{m}$ additional to the clear antinode at position $-1.6 \mu \mathrm{m}$ [see open dots and arrows in Fig. 10(b)]. By further investigating the structure of the edge mode at $\mu_{0} H_{\text {bias }}=80 \mathrm{mT}$ we found additional evidence for a two antinode mode shown in Fig. 11 (arrows and solid dots).

\section{SUMMARY}

In conclusion, the spin excitations of 4 micron permalloy disks accessible to in-plane excitation have been observed with inductive and spatially resolved techniques. We image eigenmodes of this system in zero field as well as in applied in-plane bias fields. By means of spatially resolved ferromagnetic resonance scanning Kerr microscopy radial eigenmodes up to the fifth order were directly observed. The investigation of the modal spectrum as a function of a magnetic bias field reveals localized modes in the longitudinal and transversal direction. The longitudinal localization was found to result from strong inhomogeneous internal field at the edges causing localized modes. Generally, the agreement between the experiment and the micromagnetic simulations was found to be very good.

\section{ACKNOWLEDGMENTS}

The authors would like to thank Christoph Quitmann and Frithjof Nolting from the SIM beamline at the Swiss Light Source for the PEEM measurements. This work has been supported by the DFG through the priority program SPP1133 and through the Forschergruppe FOG370.
${ }^{1}$ W. K. Hiebert, A. Stankiewicz, and M. R. Freeman, Phys. Rev. Lett. 79, 1134 (1997).

${ }^{2}$ R. J. Hicken and J. Wu, J. Appl. Phys. 85, 4580 (1999).

${ }^{3}$ J. Jorzick, S. O. Demokritov, B. Hillebrands, B. Bartenlian, C. Chappert, D. Decanini, F. Rousseaux, and E. Cambril, Appl. Phys. Lett. 75, 3859 (1999).

${ }^{4}$ Y. Acremann, C. H. Back, M. Buess, O. Portmann, A. Vaterlaus, D. Pescia, and H. Melchior, Science 290, 492 (2000).

${ }^{5}$ S. Tamaru, J. A. Bain, R. J. M. van de Veerdonk, T. M. Crawford, M. Covington, and M. H. Kryder, J. Appl. Phys. 91, 8034 (2002).

${ }^{6}$ J. P. Park, P. Eames, D. M. Engebretson, J. Berezovsky, and P. A. Crowell, Phys. Rev. Lett. 89, 277201 (2002).

${ }^{7}$ V. Novosad, M. Grimsditch, K. Y. Guslienko, P. Vavassori, Y. Otani, and S. D. Bader, Phys. Rev. B 66, 052407 (2002).

${ }^{8}$ B. Hillebrands and K. Ounadjela, Spin Dynamics in Confined Magnetic Structures I (Springer-Verlag, Berlin, 2002).

${ }^{9}$ J. Jorzick, S. O. Demokritov, B. Hillebrands, M. Bailleul, C. Fermon, K. Y. Guslienko, A. N. Slavin, D. V. Berkov, and N. L.
Gorn, Phys. Rev. Lett. 88, 047204 (2002).

${ }^{10}$ M. B. D. Olligs and C. Fermon, Phys. Rev. Lett. 91, 137204 (2003).

${ }^{11}$ G. Gubbiotti, G. Carlotti, T. Okuno, T. Shinjo, F. Nizzoli, and R. Zivieri, Phys. Rev. B 68, 184409 (2003).

${ }^{12}$ J. P. Park, P. Eames, D. M. Engebretson, J. Berezovsky, and P. A. Crowell, Phys. Rev. B 67, 020403(R) (2003).

${ }^{13}$ M. Belov, Z. Liu, R. D. Sydora, and M. R. Freeman, Phys. Rev. B 69, 094414 (2004).

${ }^{14}$ C. Bayer, J. P. Park, H. Wang, M. Yan, C. E. Campbell, and P. A. Crowell, Phys. Rev. B 69, 134401 (2004).

${ }^{15}$ L. Giovannini, F. Montoncello, F. Nizzoli, G. Gubbiotti, G. Carlotti, T. Okuno, T. Shinjo, and M. Grimsditch, Phys. Rev. B 70, 172404 (2004).

${ }^{16}$ S. B. Choe, Y. Acremann, A. Scholl, A. Bauer, A. Doran, J. Stöhr, and H. A. Padmore, Science 304, 420 (2004).

${ }^{17}$ M. Buess, R. Höllinger, T. Haug, K. Perzlmaier, U. Krey, D. Pescia, M. R. Scheinfein, D. Weiss, and C. H. Back, Phys. Rev. Lett. 93, 077207 (2004). 
${ }^{18}$ X. Zhu, M. Malac, Z. Liu, H. Qian, V. Metlushko, and M. R. Freeman, Appl. Phys. Lett. 86, 262502 (2005).

${ }^{19}$ J. Raabe, C. Quitmann, C. H. Back, F. Nolting, S. Johnson, and C. Buehler, Phys. Rev. Lett. 94, 217204 (2005).

${ }^{20}$ K. Perzlmaier, M. Buess, C. H. Back, V. E. Demidov, B. Hillebrands, and S. O. Demokritov, Phys. Rev. Lett. 94, 057202 (2005).

${ }^{21}$ T. Gerrits, H. A. M. van den Berg, J. Hohlfeld, L. Bär, and T. Rasing, Nature (London) 418, 509 (2002).

${ }^{22}$ H. W. Schumacher, C. Chappert, P. Crozat, R. C. Sousa, P. P. Freitas, J. Miltat, J. Fassbender, and B. Hillebrands, Phys. Rev. Lett. 90, 017201 (2003).

${ }^{23}$ H. W. Schumacher, C. Chappert, R. C. Sousa, P. P. Freitas, and J. Miltat, Phys. Rev. Lett. 90, 017204 (2003).

${ }^{24}$ G. N. Kakazei, P. E. Wigen, K. Y. Guslienko, V. Novosad, A. N. Slavin, V. O. Golub, N. A. Lesnik, and Y. Otani, Appl. Phys. Lett. 85, 443 (2004).

${ }^{25}$ U. Ebels, L. D. Buda, K. Ounadjela, and P. E. Wigen, in Spin Dynamics in Confined Magnetic Structures I, edited by B. Hillebrands and K. Ounadjela (Springer-Verlag, Berlin, 2002).

${ }^{26}$ M. R. Freeman, W. K. Hiebert, and A. Stankiewicz, J. Appl. Phys. 83, 6217 (1998).

${ }^{27}$ V. E. Demidov, S. O. Demokritov, B. Hillebrands, and M. Laufenberg, Appl. Phys. Lett. 85, 2866 (2004).

${ }^{28}$ S. O. Demokritov and B. Hillebrands, in Spin Dynamics in Confined Magnetic Structures I, edited by B. Hillebrands and K. Ounadjela (Springer-Verlag, Berlin, 2002).

${ }^{29}$ C. Kittel, Phys. Rev. 73, 155 (1948).

${ }^{30}$ M. Sparks, Solid State Commun. 8, 731 (1970).

${ }^{31}$ M. Sparks, Ferromagnetic Relaxation Theory (McGraw-Hill, New York, 1966).

${ }^{32}$ G. T. Rado and J. R. Weertmann, J. Phys. Chem. Solids 11, 315 (1959).

${ }^{33}$ K. Y. Guslienko, S. O. Demokritov, B. Hillebrands, and A. N. Slavin, Phys. Rev. B 66, 132402 (2002).

${ }^{34}$ T. L. Gilbert, Phys. Rev. 100, 1243 (1955).

${ }^{35} \mathrm{M}$. R. Scheinfein, details concerning the LLG code can be found at http://llgmicro.home.mindspring.com/
${ }^{36}$ I. Neudecker, G. Woltersdorf, B. Heinrich, T. Okuno, G. Gubbiotti, and C. H. Back (unpublished).

${ }^{37}$ J. Raabe, R. Pulwey, R. Sattler, T. Schweinböck, J. Zweck, and D. Weiss, J. Appl. Phys. 88, 4437 (2000).

${ }^{38}$ T. Shinjo, T. Okuno, R. Hassdorf, K. Shigeto, and T. Ono, Science 289, 930 (2000).

${ }^{39}$ A. Wachowiak, J. Wiebe, M. Bode, O. Pietzsch, M. Morgenstern, and R. Wiesendanger, Science 298, 577 (2002).

${ }^{40}$ M. Buess, Y. Acremann, A. Kashuba, C. H. Back, and D. Pescia, J. Phys.: Condens. Matter 15, R1093 (2003).

${ }^{41}$ M. Buess, T. P. J. Knowles, R. Höllinger, T. Haug, U. Krey, D. Weiss, D. Pescia, M. R. Scheinfein, and C. H. Back, Phys. Rev. B 71, 104415 (2005).

${ }^{42}$ K. Y. Guslienko, W. Scholz, R. W. Chantrell, and V. Novosad, Phys. Rev. B 71, 144407 (2005).

${ }^{43}$ B. A. Ivanov and C. E. Zaspel, Phys. Rev. Lett. 94, 027205 (2005).

${ }^{44}$ R. Zivieri and F. Nizzoli, Phys. Rev. B 71, 014411 (2005).

${ }^{45}$ M. Buess, T. Haug, M. R. Scheinfein, and C. H. Back, Phys. Rev. Lett. 94, 127205 (2005).

${ }^{46}$ A. A. Thiele, Phys. Rev. Lett. 30, 230 (1973).

${ }^{47}$ B. E. Argyle, E. Terrenzio, and J. C. Slonczewski, Phys. Rev. Lett. 53, 190 (1984).

${ }^{48}$ J. P. Park and P. A. Crowell, Phys. Rev. Lett. 95, 167201 (2005).

${ }^{49}$ A. Puzic et al., J. Appl. Phys. 97, 704 (2005).

${ }^{50}$ D. Chen, J. A. Brug, and R. B. Goldfarb, IEEE Trans. Magn. 27, 3601 (1991).

${ }^{51}$ G. Gubbiotti, O. Kazakova, G. Carlotti, M. Hanson, and P. Vavassori, IEEE Trans. Magn. 39, 2750 (2003).

${ }^{52}$ Object Oriented MicroMagnetic Framework. The OOMMF code can be found at http://math.nist.gov/oommf/

${ }^{53}$ R. Damon and J. Eshbach, J. Appl. Phys. 31, 104S (1960).

${ }^{54}$ C. Bayer, S. O. Demokritov, B. Hillebrands, and A. N. Slavin, Appl. Phys. Lett. 82, 607 (2003).

${ }^{55}$ S. O. Demokritov, B. Hillebrands, and A. N. Slavin, Phys. Rep. 348, 441 (2001). 